\title{
UTILIZAÇÃO DA INTERNET NAS ATIVIDADES DE MONITORAMENTO AMBIENTAL EM EMPRESAS DE PEQUENO E MÉDIO PORTE
}

\author{
Patricia V. de Castro Krakauer \\ Mestranda em Administração pela Universidade de São Paulo - USP \\ E-mail: pkrakauer@terra.com.br (Brasil)
}

\section{Martinho Isnard Ribeiro de Almeida}

Doutor em Administração pela Universidade de São Paulo - USP

Professor da Universidade de São Paulo - USP

E-mail: martinho@usp.br (Brasil)

\section{RESUMO}

Este artigo tem como proposta compreender a importância da sistematização do monitoramento ambiental em PMEs (pequenas e médias empresas), considerando que o entendimento de como as empresas buscam e utilizam informações da Internet é de grande relevância para esse processo, mantendo-as mais competitivas no seu mercado. Foi realizada uma revisão bibliográfica sobre monitoramento ambiental, avaliando que vários modelos genéricos são descritos na literatura, apesar de que muitos não direcionam seus esforços para empresas de pequeno ou médio porte. A metodologia utilizada foi descritiva com estudo de caso único, de forma a apresentar os procedimentos realizados por uma empresa ao utilizar dados da Internet em seu monitoramento ambiental, comparando a prática utilizada com as etapas do modelo descrito na literatura. Os resultados demonstraram que a Internet é utilizada como uma importante fonte para o monitoramento ambiental dessa empresa, mas a busca por informações on-line ocorre sem sistematização, intuitivamente e de forma reativa. Este artigo contribui para que PMEs vislumbrem a importância de monitorar o ambiente e passem a adotar um modelo formal que sistematize a obtenção e o uso da informação on-line, tornando-as mais estratégicas para o seu negócio.

Palavras-chave: Pequenas e médias empresas; Monitoramento ambiental; Internet. 


\section{INTRODUÇÃO}

Apesar da crescente importância das pequenas empresas na economia brasileira, ainda é alto o índice de mortalidade dessas empresas, sendo que um dos principais fatores citados para essa ocorrência diz respeito à falta de um planejamento formal (Dornelas, 2008) e à limitada visão do que acontece em seu ambiente (Pearce II, Chapman, \& David, 1982).

Monitoramento ambiental é um assunto abordado em vários estudos acadêmicos, sendo definido por Pearce II et al. (1982) como uma maneira organizada de observar o mundo além da empresa, de forma a antecipar oportunidades e prever perigos. Cancellier, Almeida e Estrada (2005) citam que, apesar de os estudos relacionados a essa atividade ainda serem mais direcionados a empresas de grande porte, o monitoramento ambiental tem muito a contribuir com pequenas e médias empresas.

É certo que todas as empresas praticam algum tipo de monitoramento ambiental, não sendo, muitas vezes, formais e sistemáticos em pequenas e médias empresas (PMEs). Autores como Almeida e Moreira (2004) e Pearce et al. (1982) abordam a necessidade de se estabelecer estudos específicos no que se refere à utilização de modelos simplificados que possam sistematizar o monitoramento ambiental nas empresas de menor porte, em função das suas particularidades.

Diversas são as dificuldades encontradas por pequenas e médias empresas ao buscar, analisar e monitorar informações. Falta de recursos, de tempo e a atitude dos proprietários são os principais fatores relacionados à ausência de sistematização dessas técnicas, conforme citam Almeida e Menezes (1997).

Dessa forma, a alta disponibilidade de acesso a informações que podem ser obtidas na Internet, de forma simples, rápida e gratuita, vem ao encontro da necessidade de pequenas e médias empresas, que podem utilizar esses dados para alavancar o seu monitoramento ambiental, tornando-se mais competitivas em seu mercado.

Contrariando essa aparente facilidade, apenas buscar informações não é suficiente para garantir a utilidade das mesmas para a empresa. Para Choo (2002), a informação precisa ser transformada em conhecimento para ser estratégica e relevante para a organização.

Para dados obtidos na Internet, a seleção de fontes e de variáveis é essencial em função da abundância e diversidade de informações. Choo (2000) considera que a estrutura funcional da Web, da mesma forma que facilita, restringe a busca por informações, pelo excesso de dados e de fontes. Portanto, compreender a necessidade da informação e a utilidade que a mesma terá no contexto empresarial é uma etapa fundamental para o processo de monitoramento ambiental. McGee e Prusak 
(1998) consideram, inclusive, que identificar a necessidade de informação é a etapa mais importante desse processo, sendo que muitas vezes é uma etapa não considerada pelas empresas.

Acredita-se que PMEs em geral não buscam a informação do seu ambiente de forma planejada e, portanto, não avaliam quais variáveis devem ser investigadas para que os dados obtidos sejam relevantes ao seu negócio. Agem de forma intuitiva, buscando mais informações operacionais do que estratégicas e realizando tal prática, em geral, reativamente.

Mesmo com a maior facilidade de adquirir informações na Internet, ainda falta sistematização dos processos para que as informações obtidas virtualmente se transformem em ferramentas que possam auxiliar o estabelecimento de estratégias. Benczúr (2005) cita que navegar na Internet em busca de dados não significa que a empresa esteja monitorando o seu ambiente estrategicamente, pois em geral essa atitude tende a resolver questões apenas pontuais.

A utilização da Internet para o monitoramento ambiental de PMEs ainda é um desafio, pois, apesar de utilizarem esse canal para obter uma grande variedade de informação do seu ambiente, elas ainda o fazem de forma não sistemática, sem planejamento e apenas pontualmente, conforme cita Huang (2009), o que segundo o autor resulta em decisões estratégicas de baixa qualidade.

Diante desse contexto, este estudo procura descrever as práticas utilizadas para o monitoramento ambiental de informações obtidas na Internet por pequenas e médias empresas. De forma específica, buscou-se comparar a prática levantada através do estudo de caso com as etapas do modelo prescritivo selecionado na literatura, de modo a verificar facilidades e limitações da utilização de tais modelos.

A análise proposta contribui para que PMEs vislumbrem a importância de monitorar o ambiente formalmente, podendo adotar um modelo que sistematize essa técnica, tornando as informações obtidas na Internet mais estratégicas para o seu negócio. Segundo Savioz e Blum (2002), antecipar as informações do ambiente é estrategicamente muito valioso para as empresas de menor porte. Acrescentam que as informações monitoradas são valiosas para a tomada de decisão estratégica.

$\mathrm{O}$ atual estudo está estruturado em cinco capítulos. Neste primeiro capítulo, apresenta-se o contexto do tema pesquisado, bem como os objetivos propostos para o estudo. No segundo, apresentam-se alguns aspectos que favorecem a conceituação de PMEs e informações on-line, bem como a revisão teórica sobre modelos prescritivos descritos na literatura de forma a servir de alicerce para a pesquisa de campo. No capítulo 3, são apresentadas as etapas metodológicas. O quarto descreve as atividades de monitoramento de uma PME à luz da fundamentação teórica e o último aborda as considerações finais sobre os achados, as limitações metodológicas e sugestões para estudos futuros. 


\section{FUNDAMENTAÇÃO TEÓRICA}

\subsection{DEFINIÇÃO DE PEQUENAS E MÉDIAS EMPRESAS (PMES)}

Almeida e Moreira (2004) citam a dificuldade em definir o porte da empresa, em função de que vários são os parâmetros que podem ser considerados nessa classificação, como: características específicas, receita bruta anual e número de colaboradores.

Benczúr (2005), em sua pesquisa relacionada ao monitoramento do ambiente, considerou três aspectos relevantes para definir o tamanho da empresa: número de níveis hierárquicos, número de colaboradores e resultados em vendas.

No Boletim Estatístico do Sebrae (2005) encontra-se a definição relacionada ao porte da empresa e o BNDES (2002) as classifica de acordo com a renda operacional bruta anual, conforme quadro 1.

\begin{tabular}{l|l|l}
\multicolumn{1}{c|}{ Porte da Empresa } & \multicolumn{1}{c|}{ SEBRAE (2005) } & \multicolumn{1}{c}{ BNDES (2002) } \\
\hline Microempresa & Até 9 empregados & Inferior ou igual a R\$ 1.200 mil. \\
\hline Pequena Empresa & De 10 a 49 empregados & Entre $\mathrm{R} \$ 1.200$ mil e $\mathrm{R} \$ 10.500$ mil \\
\hline Média Empresa & De 50 a 99 empregados & Entre $\mathrm{R} \$ 10.500$ mil e $\mathrm{R} \$ 60$ milhões. \\
\hline Grande Empresa & Mais de 99 empregados & Acima de $\mathrm{R} \$ 60$ milhões. \\
\hline
\end{tabular}

Quadro 1 - Classificação do porte de empresas de comércio e serviço.

Fonte: Elaborado pelos autores.

Características e particularidades também podem ser consideradas ao se classificar uma empresa como PME, conforme citam Almeida e Moreira (2004). Contudo, no presente estudo para definir uma empresa como PME, serão considerados os critérios apresentados no Quadro 1, uma vez que características específicas são subjetivas e de difícil mensuração. Considerou-se, portanto, que o número de colaboradores e a renda permitem maior transparência e facilidade para a classificação.

\subsection{BUSCA DE INFORMAÇÕES ON-LINE}

Nonaka e Takeuchi (1997) conceituam informação como um fluxo de mensagens inseridas em um determinado contexto, sendo um meio para se construir o conhecimento. McGee e Prusak (1998) consideram que a informação é um importante recurso estratégico das organizações, sendo dados coletados, organizados e com significado, gerenciados de tal forma a favorecer vantagem competitiva.

A busca por informações sofreu mudanças com o advento da Internet, que vem sendo utilizada como uma importante ferramenta empresarial, alavancando novas oportunidades para vários tipos de

Revista de Administração e Inovação, São Paulo, v. 9, n. 1, p.15-30, jan./mar. 2012. 
negócios. Turban, Rainer e Potter (2005) citam que, com a expansão da Internet, aumentaram os benefícios para as organizações, para os indivíduos e para a sociedade como um todo, com acesso fácil e rápido a todo tipo de informação.

Contudo, Fidelis (2001) cita que a facilidade de se obter dados esconde a dificuldade de encontrar informações que sejam realmente úteis. Essa característica faz com que a sistematização de um modelo que formalize a utilização das informações on-line seja fundamental para que estas se tornem estratégicas e não apenas informações pontuais, sem uma visão do futuro.

Deve-se ressaltar que uma das vantagens da Internet é a sua rápida capacidade de atualização, um fato que deve ser considerado ao avaliar esse canal como fonte de informação. Fidelis (2001) considera que, apesar das desvantagens, os benefícios são valiosos, como: maior número de fontes para consulta de dados, baixo custo para obtenção de informações, conforto e economia de tempo.

Para Huang (2009), as empresas de menor porte buscam as informações informalmente, não possuindo uma sistemática para esse processo, estando muito vinculadas à figura do seu proprietário, cujas características interferem no processo.

As vantagens das informações on-line são importantes para PMEs que possuem limitação de recursos financeiros e de tempo para monitorar o seu ambiente. As informações on-line, bem selecionadas e analisadas, podem se transformar em valiosas ferramentas competitivas.

\subsection{MODELOS DE MONITORAMENTO AMBIENTAL}

Vários modelos genéricos são descritos na literatura, sendo que a maior parte não direciona seus esforços para empresas de pequeno ou médio porte. Cancellier et al. (2005) citam a importância de se considerar as particularidades de pequenas empresas no monitoramento ambiental, inclusive propondo a simplificação de modelos para a sua aplicação prática. Os autores analisam modelos prescritivos propostos por Aaker (1983), Calori (1989) e Pearce II et al., propondo uma síntese em seis passos, apresentada no Quadro 2.

McGee e Prusak (1998) salientam que a informação facilita o estabelecimento de estratégias quando revelam oportunidades e ameaças originadas das mudanças e evolução do ambiente externo. O modelo proposto pelos autores sugere seis etapas para o gerenciamento da informação.

Aaker (1983) propôs um modelo simplificado com seis etapas, favorecendo a flexibilidade e podendo ser implantado com baixo custo para que as empresas possam utilizá-lo com mais frequência e deixem, assim, de perder valiosas informações.

No Quadro 2 apresentam-se as etapas desses três modelos prescritivos descritos na literatura:

Revista de Administração e Inovação, São Paulo, v. 9, n. 1, p.15-30, jan./mar. 2012. 


\begin{tabular}{|c|c|c|c|}
\hline Etapas & \multicolumn{3}{|c|}{ Modelos } \\
\hline & McGee e Prusak (1998) & Aaker (1983) & Cancellier et al. (2005) \\
\hline 1 & $\begin{array}{l}\text { Identificação de } \\
\text { necessidades/requisitos } \\
\text { de informação. }\end{array}$ & $\begin{array}{l}\text { Conhecimento da necessidade de } \\
\text { informação. }\end{array}$ & $\begin{array}{l}\text { Definição das variáveis e } \\
\text { necessidades de informação. }\end{array}$ \\
\hline 2 & Coleta de informações. & Definição das fontes. & Identificação de fontes. \\
\hline 3 & $\begin{array}{l}\text { Classificação e } \\
\text { armazenamento de } \\
\text { informação. }\end{array}$ & Seleção dos participantes. & Definição dos responsáveis. \\
\hline 4 & $\begin{array}{l}\text { Desenvolvimento de } \\
\text { produtos e serviços de } \\
\text { informação. }\end{array}$ & Atribuição de tarefas. & Armazenamento. \\
\hline 5 & $\begin{array}{l}\text { Distribuição e } \\
\text { disseminação da } \\
\text { informação. }\end{array}$ & $\begin{array}{l}\text { Armazenamento/processamento } \\
\text { de dados. }\end{array}$ & Processamento. \\
\hline 6 & $\begin{array}{l}\text { Análise e uso da } \\
\text { informação. }\end{array}$ & Disseminação das informações. & Divulgação. \\
\hline
\end{tabular}

Quadro 2 - Etapas dos modelos de monitoramento ambiental.

Fonte: Elaborado pelos autores.

Diante desse contexto, para o presente estudo será considerada a síntese apresentada por Cancellier et al. (2005), por estar adaptada a realidade de PMEs, sendo utilizado o modelo de segmentação ambiental proposto por Almeida (2009) para a seleção de variáveis de forma a estruturar o processo de monitoramento.

\subsection{SELEÇÃO DE VARIÁVEIS}

Definir quais informações são relevantes é uma etapa fundamental para a sistematização do monitoramento ambiental de empresas de qualquer porte, em especial de pequenas e médias, que, com menos recursos, podem concentrar seus esforços no que é mais estratégico para o seu negócio. Xavier e Cancellier (2008) citam que a seleção de variáveis é necessária para estruturar o monitoramento ambiental, sendo a primeira etapa desse processo.

Para Almeida (2009), é fundamental definir as variáveis ambientais que influenciam a empresa, facilitando a coleta de informações, não gerando nesse processo um número excessivo de dados. O autor propõe um modelo que divide o ambiente em quatro segmentos, a saber: macroambiente clima, macroambiente solo, ambiente operacional e ambiente interno, sendo que diferentes variáveis ambientais são relacionadas em cada um dos segmentos, conforme Quadro 3. 


\begin{tabular}{|c|c|}
\hline $\begin{array}{l}\text { Macroambiente } \\
\text { Clima }\end{array}$ & $\begin{array}{l}\text { Variáveis relacionadas às ações do governo, como: inflação, crescimento do PIB, } \\
\text { legislação. }\end{array}$ \\
\hline $\begin{array}{l}\text { Macroambiente } \\
\text { Solo }\end{array}$ & $\begin{array}{l}\text { Variáveis referentes à população e suas características, como: crescimento } \\
\text { populacional, por região, por sexo e por faixa de renda. }\end{array}$ \\
\hline $\begin{array}{l}\text { Ambiente } \\
\text { Operacional }\end{array}$ & Variáveis relacionadas a concorrentes, fornecedores, clientes e tecnologia. \\
\hline $\begin{array}{l}\text { Ambiente } \\
\text { Interno }\end{array}$ & $\begin{array}{l}\text { Valores e aspirações dos proprietários e colaboradores, analisando a cultura da } \\
\text { empresa. }\end{array}$ \\
\hline
\end{tabular}

\subsection{FONTES DE INFORMAÇÕES}

McGee e Prusak (1998) citam a importância de se definir fontes variadas de informações, sendo que para essa seleção deve-se considerar que a coleta das informações deve ser realizada com criatividade e disponibilizada em tempo hábil.

Aaker (1983) sugere que sejam priorizadas fontes com as quais a empresa já tenha familiaridade, facilitando o processo de monitoramento. Com relação a pequenas empresas, Pearce II et al. (1982) salientam que, além de se utilizar fontes habituais, deve ser considerada a facilidade de acesso.

\subsection{ATRIBUIÇÃO DE RESPONSABILIDADES}

Sobre a definição de responsabilidades, tanto os autores do modelo selecionado quanto Aaker (1983) e Pearce II et al. (1982) citam que o monitoramento deve ser descentralizado, envolvendo no processo o maior número possível de pessoas. Essa descentralização deve, contudo, considerar o perfil dos participantes, sendo selecionadas atividades de acordo com a função e interesse de cada colaborador (Pearce II et al., 1982).

Aaker (1983) sugere que tenham prioridade as pessoas mais expostas a fontes de informações, bem como aquelas envolvidas diretamente com o planejamento estratégico ou áreas afins.

\subsection{ARMAZENAMENTO}

Xavier e Cancellier (2008) descrevem a importância do armazenamento nos processos de monitoramento, que envolve não apenas a guarda física das informações, mas também a sua classificação e mecanismos de recuperação. McGee e Prusak (1998) também citam a necessidade de se 
classificar a informação de forma a facilitar o acesso e propõem a utilização de índices on-line ou impressos, conforme as características de acessibilidade à Internet da empresa.

Aaker (1983) sugere que as informações podem ser armazenadas em simples arquivos ou utilizando-se sofisticados sistemas de informação. O mais importante, contudo, é a facilidade de acesso às informações e a utilização de um local único, de forma que importantes dados não sejam perdidos.

\subsection{ANÁLISE DAS INFORMAÇÕES}

Para Choo (2002), a capacidade de identificar oportunidades depende da coragem de observar o futuro, sendo a análise e interpretação das informações do ambiente fundamentais para a saúde das organizações.

McGee e Prusak (1998) consideram que é o uso da informação que faz com que o processo de monitoramento influencie a tomada de decisões e o desenvolvimento estratégico das empresas.

Aaker (1983) sugere que a análise das informações monitoradas deve contar com o apoio de especialistas, o que pode ser inviável para PMEs, conforme opinião de Xavier e Cancellier (2008), em função do custo e do reduzido número de colaboradores. Salientam ainda o tempo como limitante nessa etapa do processo, pois, dependendo da variável monitorada, as informações podem ficar obsoletas.

No modelo selecionado para o atual estudo, verifica-se uma forma de análise adequada à realidade de pequenas e médias empresas, consistindo no cruzamento de diferentes informações e discussões regulares entre os dirigentes da empresa.

\subsection{DIVULGAÇÃO}

A disseminação das informações pode ocorrer junto com as discussões de análise, sendo que as duas etapas acabam se fundindo, segundo os autores do modelo selecionado.

Aaker (1983) cita que nessa etapa devem ser selecionados os canais mais adequados para a disseminação das informações, podendo variar entre arquivos, resumos, relatórios e sistemas informatizados.

McGee e Prusak (1998) comentam que deve haver um grupo responsável pela disseminação, sendo fundamental a elaboração de ações que incentivem o acesso e uso das informações por todos os colaboradores. 


\section{METODOLOGIA}

Para se atingir o objetivo proposto, optou-se por uma pesquisa descritiva, utilizando-se como abordagem metodológica um estudo de caso único. Justifica-se essa decisão em função da necessidade de se delinear (Malhotra, 2006) os procedimentos realizados por uma empresa ao utilizar dados da Internet em seu monitoramento ambiental, analisando-se as etapas utilizadas nessa atividade. A seleção da empresa foi feita por conveniência, considerando-se tanto a facilidade de acesso aos dados quanto a contribuição com o propósito da pesquisa, uma vez que a empresa selecionada apresentou elementos significativos de forma a clarificar o problema apresentado.

Para Eisenhardt (1989), o estudo de caso permite a análise em profundidade de uma situação em particular, identificando variáveis e suas inter-relações que poderiam não ser percebidas através de outro método. A opção de investigar exclusivamente uma empresa foi baseada na argumentação de Yin (2005), que ressalta que o caso único pode ser utilizado na avaliação e explanação de teorias, o que condiz com a proposta do presente estudo, que pretende comparar a prática utilizada no monitoramento ambiental de PMEs com modelos descritos na literatura.

A pesquisa de campo foi realizada na empresa Ramo Sistemas, atuante no setor de tecnologia há 28 anos. Atualmente possui 49 funcionários, o que a caracteriza como uma PME, conforme apresentado no Quadro 1, item 2.1.

A empresa, estabelecida em São Paulo e com filial nos Estados Unidos, desenvolve, vende e implanta sistemas integrados de gestão (ERP), além de serviços específicos de consultoria técnica. Sua estrutura é composta por quatro divisões: marketing e vendas, técnico, administrativo e suporte ao cliente. Procurou-se entrevistar, além de um dos proprietários, o responsável por cada departamento, buscando-se obter uma visão mais ampla das atividades de monitoramento e as diferentes necessidades das áreas, conforme Quadro 4.

Foram utilizados dados secundários, obtidos através da documentação da empresa, e dados primários, coletados através de entrevistas semiestruturadas, individuais, realizadas em novembro de 2009, com duração de cerca de 1 hora cada. Apenas a entrevista com o proprietário (E1) foi gravada, em virtude da falta de autorização dos demais entrevistados. A documentação analisada focou-se nas informações obtidas na Internet, em função do escopo do estudo. 
Utilização da internet nas atividades de monitoramento ambiental em empresas de pequeno e médio porte

\begin{tabular}{c|c|c|c}
\hline Entrevistado & Função & $\begin{array}{c}\text { Tempo na } \\
\text { empresa }\end{array}$ & $\begin{array}{c}\text { Tempo no } \\
\text { mercado }\end{array}$ \\
\hline E1 & Diretor Geral (sócio) & 19 anos & 22 anos \\
\hline E2 & Gerente Operacional & 13 anos & 20 anos \\
\hline E3 & Gerente Desenvolvimento & 13 anos & 17 anos \\
\hline E4 & Gerente Suporte ao Cliente & 6 anos & 8 anos \\
\hline E5 & Coordenação Administrativa & 9 anos & 9 anos \\
\hline
\end{tabular}

Quadro 4 - Classificação dos entrevistados.

Fonte: Elaborado pelos autores.

O roteiro elaborado para as entrevistas foi desenvolvido em três seções, sendo que as perguntas abordaram questões relacionadas à utilização da Internet como fonte de informação, seleção de variáveis ambientais e a sistematização para obtenção, análise e utilização das informações.

Os dados obtidos foram consolidados e analisados qualitativamente conforme as etapas descritas por Bauer e Gaskell (2007): transcrição, classificação e análise. Posteriormente os dados primários foram cruzados com os secundários, de forma a possibilitar possíveis acréscimos nos achados.

\section{ANÁLISE DE RESULTADOS}

O principal resultado observado com os dados obtidos foi a análise da sistemática utilizada para o monitoramento ambiental de informações obtidas na Internet pela empresa estudada. Tal sistemática foi analisada através dos achados da pesquisa de campo e com a comparação da prática realizada pela empresa com o modelo prescritivo selecionado na literatura, de forma a verificar facilidades e limitações da utilização de modelos formais.

Com a análise dos achados e o cruzamento com os dados secundários, observou-se que a empresa está atenta ao seu ambiente e procura monitorá-lo conforme a necessidade de cada departamento, principalmente em função do dinamismo do setor tecnológico. Benczúr (2005) comenta que o quanto mais instável é o ambiente em que a empresa está inserida, maior a necessidade de monitorá-lo, como é o caso de empresas de tecnologia da informação.

A volatilidade do ambiente tecnológico é uma preocupação constante dos dirigentes, que procuram monitorar essa variável de forma a estarem atualizados com novas tendências, buscando competitividade, prevendo oportunidades e ameaças futuras.

A Internet é a principal fonte de busca das informações que a empresa necessita, sendo que essa busca em geral acontece de forma reativa ao aparecimento de problemas ou necessidades. Apenas um entrevistado (E3) citou que realiza essa busca trimestralmente, de forma regular.

Revista de Administração e Inovação, São Paulo, v. 9, n. 1, p.15-30, jan./mar. 2012. 
Apesar de não utilizar uma metodologia que sistematize o monitoramento, várias etapas do modelo teórico são utilizadas pela empresa de forma intuitiva, porém sem formalização, conforme apresentado no Quadro 5.

\begin{tabular}{l|c|c|c|c|c}
\hline \multicolumn{1}{c|}{ Etapa } & \multicolumn{5}{c}{ Entrevistado } \\
& E1 & E2 & E3 & E4 & E5 \\
\hline Seleção de variáveis & $\mathrm{X}$ & $\mathrm{X}$ & $\mathrm{X}$ & $\mathrm{X}$ & $\mathrm{X}$ \\
\hline Definição de fontes & $\mathrm{X}$ & $\mathrm{X}$ & $\mathrm{X}$ & $\mathrm{X}$ & $\mathrm{X}$ \\
\hline Indicação de responsabilidades & & & & & \\
\hline Armazenamento & & $\mathrm{X}$ & $\mathrm{X}$ & & $\mathrm{X}$ \\
\hline Análise & $\mathrm{X}$ & $\mathrm{X}$ & $\mathrm{X}$ & $\mathrm{X}$ & \\
\hline Disseminação & $\mathrm{X}$ & & $\mathrm{X}$ & & \\
\hline
\end{tabular}

Quadro 5 - Etapas do modelo de monitoramento realizadas pelos entrevistados.

Fonte: Elaborado pelos autores.

Mesmo realizando o monitoramento intuitivamente, todos os entrevistados consideraram que essa atividade é importante tanto para a empresa quanto para o profissional, que pode se manter atualizado e preparado para o futuro.

\subsection{SELEÇÃO DE VARIÁVEIS}

Na empresa analisada, cada departamento monitora a variável que mais influencia o seu setor, sendo uma decisão intuitiva do responsável pela área, não havendo uma análise conjunta com os demais dirigentes da empresa.

Observa-se que há uma maior atenção com o ambiente operacional, sendo que variáveis do macroambiente solo e do ambiente interno não são monitoradas. Com relação ao ambiente interno, pode-se citar que, apesar de não monitoradas, há uma preocupação dos proprietários com os aspectos internos da empresa, como variáveis relativas à formação e capacitação dos funcionários, conforme pode ser observado pelo comentário do entrevistado 1 (E1): “Hoje em dia temos que estar atentos às necessidades dos funcionários, no que diz respeito à sua formação profissional e técnica, senão os perdemos".

O Quadro 6 apresenta as variáveis que são monitoradas, classificadas conforme o modelo de segmentação ambiental (Almeida, 2009). 
Utilização da internet nas atividades de monitoramento ambiental em empresas de pequeno e médio porte

\begin{tabular}{l|l|c|c|c|c|c}
\hline \multicolumn{1}{c|}{ Variável } & \multicolumn{5}{c}{ Entrevistado que monitora } \\
\hline \multicolumn{1}{c|}{} & & E1 & E2 & E3 & E4 & E5 \\
\hline Clima & Legislação & $\mathrm{X}$ & $\mathrm{X}$ & $\mathrm{X}$ & $\mathrm{X}$ & $\mathrm{X}$ \\
\hline Clima & Indicadores Econômicos & & $\mathrm{X}$ & & $\mathrm{X}$ & $\mathrm{X}$ \\
\hline Operacional & Salários do Mercado & $\mathrm{X}$ & $\mathrm{X}$ & & & \\
\hline Operacional & Concorrência & $\mathrm{X}$ & & $\mathrm{X}$ & & \\
\hline Operacional & Clientes Perspectiva & $\mathrm{X}$ & $\mathrm{X}$ & & & \\
\hline Operacional & Fornecedores & $\mathrm{X}$ & & & & \\
\hline Operacional & Novas Tecnologias & $\mathrm{X}$ & $\mathrm{X}$ & $\mathrm{X}$ & $\mathrm{X}$ & \\
\hline Operacional & Oportunidades de financiamentos & $\mathrm{X}$ & & & & \\
\hline
\end{tabular}

Quadro 6 - Variáveis monitoradas pela empresa analisada.

Fonte: Elaborado pelos autores.

A legislação é uma variável monitorada por todos os departamentos da empresa, principalmente em função de que modificações nessa variável podem significar mudanças no produto. Outra variável importante é a que se refere a novas tecnologias, sendo que cada departamento monitora o aspecto mais relevante ao seu setor. Conforme citam Xavier e Cancellier (2008), novas tecnologias, como lançamentos e alterações em produtos que possam modificar os serviços prestados pela empresa, devem ser monitoradas de perto.

Pelo apresentado, vislumbra-se que a seleção de variáveis acontece em função da necessidade pontual e não de forma prévia, apesar de considerarem a importância dessa atitude, conforme também citado por Aaker (1983) e Pearce II et al. (1982).

\subsection{FONTES DE INFORMAÇÃO}

Uma importante etapa para o monitoramento de informações on-line é a seleção das fontes que serão utilizadas para a busca de informações, em função da diversidade e do grande número de possibilidades existente na Internet (Choo, 2000).

A empresa estudada utiliza em geral a Internet para buscar informações para monitorar o seu ambiente, principalmente em função do baixo custo e da capacidade de rápida atualização desse canal, benefício fundamental para um setor em constante mudança.

Existe uma preocupação com a fidedignidade da fonte on-line, preferindo-se fontes oficiais do governo, publicações de jornais on-line e sites de parceiros, fornecedores e de associações. Utilizam também outros canais, um número menor de vezes, como publicações especializadas, convenções nacionais e internacionais e informações de mercado obtidas pelo setor de vendas e atendimento ao cliente.

\subsection{ATRIBUIÇÃO DE RESPONSABILIDADES}

Revista de Administração e Inovação, São Paulo, v. 9, n. 1, p.15-30, jan./mar. 2012. 
Não há uma definição clara sobre a responsabilidade pelo monitoramento das informações do ambiente, sendo que o responsável pelo departamento monitora o que lhe é mais relevante. O proprietário (E1) citou que existe uma divisão informal de quem deve se preocupar com qual variável, mas que em geral a iniciativa pela busca de informações do ambiente é de sua responsabilidade.

Tal fato também foi observado no trabalho realizado por Huang (2009), que comenta que em empresas de menor porte a busca por informações e a análise das mesmas é de responsabilidade do proprietário ou do principal gestor do negócio.

\subsection{ARMAZENAMENTO E ANÁLISE}

As informações obtidas na Internet são armazenadas em arquivos eletrônicos, organizados em pastas no computador, porém sem classificação, não havendo um índice para buscas futuras. Em muitas ocasiões elas são mantidas apenas na memória dos dirigentes, conforme também citado no estudo de Cancellier et al. (2005).

A análise é feita individualmente, em geral pelo proprietário ou gerentes, mas não há uma regularidade nessa tarefa. Muitas vezes essa análise é compartilhada com profissionais de outras empresas do mesmo setor, considerados parceiros de negócios, principalmente no que se refere a inovações tecnológicas.

Em função da recente elaboração de um mapa estratégico na empresa, o entrevistado E1 acredita que essa situação deve ser alterada, sendo que estão previstas reuniões regulares para análise de diversos fatores, entre eles, variáveis do ambiente.

\subsection{DIVULGAÇÃO}

As informações on-line das variáveis monitoradas são disseminadas oralmente, porém restritas ao grupo pertencente ao departamento que a monitorou. Todos os entrevistados consideraram que deveria haver uma maior divulgação das informações obtidas sobre o ambiente organizacional, pois acreditam que muitos dados acabam sendo perdidos pela falta de compartilhamento e, conforme citam Xavier e Cancellier (2008), podem levar a ações não alinhadas estrategicamente.

\section{CONSIDERAÇÕES FINAIS}

Esta pesquisa indicou que, apesar de a empresa analisada não utilizar um modelo formal de monitoramento ambiental, várias etapas descritas na literatura são realizadas, de forma não sistemática.

Revista de Administração e Inovação, São Paulo, v. 9, n. 1, p.15-30, jan./mar. 2012. 
Observou-se que, apesar do entendimento de que o monitoramento ambiental é fundamental para o estabelecimento de estratégias futuras e para a competitividade da empresa, essa atividade é pouco estruturada na empresa estudada, sendo essa atitude já citada em outros estudos acadêmicos focados em PMEs, como o de Cancellier et al. (2005).

A maior preocupação da empresa analisada é com relação às inovações tecnológicas, aos clientes e à legislação, principalmente devido às alterações nos produtos que mudanças nessas variáveis podem sugerir. Por isso, essas variáveis são monitoradas constantemente e de forma proativa.

Apenas para o proprietário existe uma relação entre o monitoramento e o estabelecimento de estratégias, pois para os colaboradores essa atividade é importante apenas para atingir objetivos pontuais, mais relacionados a questões operacionais.

Conforme também descrito no estudo de Xavier e Cancellier (2008), os entrevistados do presente estudo não consideraram relevante monitorar o crescimento do mercado. Eles acreditam que há uma dificuldade em se monitorar um mercado instável e deixam tal atividade sob responsabilidade de fornecedores e associações.

Recomenda-se que a empresa estudada estabeleça critérios para a seleção das variáveis que devem ser monitoradas e formalize as responsabilidades pelo monitoramento, sendo que essa atribuição deve continuar descentralizada, conforme citado pelos autores do modelo teórico utilizado.

Para divulgar de forma mais ampla os resultados do monitoramento, deve-se organizar um arquivo sistemático, facilitando a consulta dos dados, e fazer reuniões periódicas para discussões sobre o monitoramento e acompanhamento da estratégia empresarial.

Interessante citar que a não utilização de uma sistemática para o monitoramento do ambiente parece estar mais relacionada à falta de um planejamento estratégico, o que é comum em PMEs, conforme citado por Dornelas (2008).

Acredita-se que ocorrerão modificações na empresa analisada em função da determinação dos dirigentes em desenvolver um mapa estratégico, o que possibilitaria uma nova averiguação no futuro, inclusive como sugestão futura a realização de uma pesquisa-ação para observar a implantação de um modelo de monitoramento ambiental em PMEs.

Importante destacar que este estudo tem limitações no que se refere à generalização dos dados, uma vez que foi utilizada uma pesquisa qualitativa com estudo de caso único, não possibilitando a extrapolação dos dados para todo o segmento de pequenas e médias empresas, mas representando uma ilustração para as PMEs que almejem monitorar as informações do seu ambiente. 


\section{REFERÊNCIAS}

Aaker, D. (1983). Organizing a strategic information scanning system. California Management Review, 25(2), 76-83.

Almeida, M. I. R. (2009). Manual de planejamento estratégico. São Paulo: Atlas.

Almeida, M. I. R., \& Menezes, E. J. C. (1997). Será possível as pequenas e médias empresas crescerem sem informações do ambiente? Anais do Encontro da Associação Nacional de PósGraduação e Pesquisa em Administração, Rio das Pedras, RJ, Brasil, 21.

Almeida, M. I. R., \& Moreira, A. L., Jr. (2004). A análise do ambiente organizacional na pequena empresa: um estudo em PME'S na cidade de São Paulo. Anais do Congresso Estrategias para el Desarrollo e Inserción Global, Camboriú, SC, Brasil, 17.

Banco Nacional de Desenvolvimento Econômico e Social. (2002). Carta circular 64. Rio de Janeiro: Autor.

Bauer, M., \& Gaskell, G. (2007). Pesquisa qualitativa com texto, imagem e som (6a ed.). Petrópolis: Vozes.

Benczúr, D. (2005). Environmental scanning: how developed is information acquisition in Western European companies? Information Research: an International Electronic Journal, 11(1), 00-00.

Calori, R. (1989). Designing a business scanning system. Long Range Planning, 22(1), 69-82.

Cancellier, E. L. P., Almeida, M. I. R., \& Estrada, R. J. S. (2005). Monitoramento do ambiente externo na pequena empresa: aplicações e limitações dos sistemas existentes. Anais do Encontro de Estudos em Estratégia, Rio de Janeiro, RJ, Brasil, 2.

Choo, C. W. (2002). Information management for the intelligent organization: the art of scanning the environment (ASIS Monograph Series). Medford, NJ: Information Today.

Choo, C. W. (2000). Information seeking and knowledge work on the world wide web. Dordrecht: Kluwer Academic Press.

Dornelas, J. C. A. (2008). Empreendedorismo: transformando idéias em negócios (3a ed.). Rio de Janeiro: Elsevier.

Eisenhardt, K. M. (1989). Building theories from case study research. Academy of Management Review, 14(4), 532-550.

Fidelis, J. R. F. (2001). A Internet como fonte de informação para inteligência empresarial: um estudo de caso em indústrias de micro, pequeno e médio portes. Dissertação de Mestrado, Universidade Federal de Minas Gerais, Belo Horizonte, MG, Brasil.

Huang, X. (2009). Strategic decision making in Chinese SMEs. Chinese Management Studies, 3(2), $87-101$.

Malhotra, N. (2006). Pesquisa de marketing: uma orientação aplicada (4a ed.). Porto Alegre: Bookman.

McGee, J., \& Prusak, L. (1998). Gerenciamento estratégico da informação: aumente a competitividade e a eficiência de sua empresa utilizando a informação como uma ferramenta estratégica. Rio de Janeiro: Campus. 
Nonaka, I., \& Keuchi, H. (1997). Criação de conhecimento na empresa (19a ed.). Rio de Janeiro: Elsevier.

Pearce II, J. A., Chapman, B. L., \& David, F. R. (1982). Environmental scanning for small and growing firms. Journal of Small Business Management, 20(3), 27-34.

Ramo Sistemas. Pesquisa geral no site. Recuperado em 30 de janeiro, 2010, de http://www.ramo.com.br.

Savioz, P., \& Blum, M. (2002). Strategic forecast tool for SMEs: how the opportunity landscape interacts with business strategy to antecipate technological trends. Technovation, 22(2), 91-100.

Serviço Brasileiro de Apoio às Micro e Pequenas Empresas. Boletim estatístico de micro e pequenas empresas: observatório SEBRAE. Brasília: Autor.

Turban, E., Rainer, R. K., \& Potter, R. E. (2005). Administração da tecnologia da informação: teoria e prática. Rio de Janeiro: Elsevier.

Xavier, W. G, \& Cancellier, E. L. P. L. (2008). Atividades de monitoramento em empresas de startup de base tecnológica na indústria do turismo. Análise, 19(2), 107-119.

Yin, R. K. (2005). Estudo de caso: planejamento e métodos. Porto Alegre: Bookman.

\title{
USING THE INTERNET IN THE ACTIVITIES OF ENVIRONMENTAL SCANNING IN SMALL AND MEDIUM ENTERPRISES
}

\begin{abstract}
This article aims to understand the importance of systematic environmental scanning in SMEs (small and medium enterprises), whereas the understanding of how companies seek and use information from the Internet is of great relevance to this process, keeping them more competitive in their market. For this was a literature review of environmental scanning, evaluating that several generic models are described in the literature, although many don't direct their efforts for small and medium enterprises. The methodology used was a descriptive single case study to present the procedures performed by a company that uses data from the Internet in its environmental scanning, comparing the practice used with the steps of the model described in the literature. The results showed that the Internet is used as an important source for environmental scanning of this company, but the search for on-line information happens without systematic, intuitively and reactively. This article contributes to help SMEs to perceive the importance of scanning the environment and begin to adopt a formal model that systematizes the search and use of on-line information, making them more strategic to their business.
\end{abstract}

Keywords: Small and medium enterprises; Environmental scanning; Internet.

Data do recebimento do artigo: 07/08/2011

Data do aceite de publicação: 18/12/2011

Revista de Administração e Inovação, São Paulo, v. 9, n. 1, p.15-30, jan./mar. 2012. 